\title{
Empleo de biocatalizadores en la degradación de material lignocelulósico: principales impactos
}

\section{Use of biocatalizers in the degradation of material lignocellulose: main impacts}

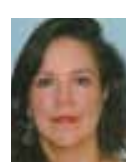

\section{Salvador Pinos Carmen}

Master Complementaire en Biotechnologie et Biologie

Docente Universidad Central del Ecuador

e-mail: casalvador@uce.edu.ec

González Erenio

Universidad Central "Marta Abreu" de Las Villas, Cuba e-mail: erenio@uclv.edu.cu

Rojas Maira

Universidad de las Américas

e-mail: maira.rojas@udla.edu.ec

\section{Mesa Leyanis}

Universidad Central "Marta Abreu" de Las Villas, Cuba e-mail: leyanis.mesa@gmail.com

\section{Batallas Fernando}

Universidad Central "Marta Abreu" de Las Villas, Cuba

e-mail: afbm@uclv.edu.cu

\section{Pérez-Martínez Amaury \\ Universidad Estatal Amazónica}

e-mail: amperez@uea.edu.ec

\section{Concepción Diana}

Universidad Central "Marta Abreu" de Las Villas, Cuba

e-mail: dianac@uclv.edu.cu

\section{Resumen}

El presente trabajo explica los esfuerzos actuales para desarrollar una tecnología accesible, rentable y limpia para el aprovechamiento de residuos lignocelulósicos para la obtención de etanol y otros derivados mediante procesos fermentativos de los diferentes azúcares y subproductos que resultan de la degradación de la celulosa. La biomasa, cuyo principal componente es la celulosa, es la materia prima más abundante del planeta, y su aprovechamiento racional produciría enormes beneficios económicos y ecológicos. La hidrólisis ácida de la celulosa es costosa y contaminante. Por lo tanto, se intenta llevar a escala industrial la hidrólisis enzimática, mediante enzimas producidas por varias especies de hongos (Trichoderma, Aspergillus, etc.), bacterias y otros organismos, y buscando otras fuentes industrialmente útiles para una biorrefinería. Es ecológicamente beneficiosa, y potencialmente mucho más económica, pero es necesario reproducir a escala industrial la actividad observada en laboratorio. La ingeniería genética ayuda a diversificar la producción de enzimas o aumentar la cantidad producida por los organismos. La hidrólisis enzimática completa usa enzimas celulolíticas: endoglucanasas, exoglucanasas y ß-glucosidasas, difícilmente producidas en cantidades industrialmente interesantes por un solo organismo, siendo necesario combinar varios de ellos. Además, técnicas como reciclaje o recirculación de enzimas dentro del biorreactor ayudarían a un aprovechamiento integral. Otra línea de investigación es la modelación matemática de la producción de los crudos enzimáticos con simuladores como Superpro Designer y otros. La gran variedad de productos obtenidos a partir de biomasa vegetal, desde etanol hasta ácido cítrico, ácido láctico, ácidos urónicos, ácido acético, etc., respaldan los beneficios económicos, sociales, industriales y ecológicos que generaría esta tecnología.

Palabras clave: enzimas; celulasas; hidrólisis enzimática; biorreactor; actividad celulolítica; biomasa

\section{Abstract}

The present work explains the current efforts to develop an accessible, profitable and clean technology for the utilization of lignocellulosic residues to obtain ethanol and other derivatives through fermentative processes of the different sugars and by-products that result from the degradation of cellulose. Biomass, whose main component is cellulose, is the most abundant raw material on the planet, and its rational use would produce enormous economic and ecological benefits. Acid hydrolysis of cellulose is expensive and pollutant. Therefore, it is intended to escalate to industrial levels the enzymatic hydrolysis of cellulose, by means of enzymes produced by several species of fungi (Trichoderma, Aspergillus, etc.), bacteria and other organisms, and looking for other industrially useful sources for a biorefinery. It is ecologically beneficial, and potencially much less expensive, but it is necessary to reproduce to an industrial scale the activity observed in laboratory conditions. Genetic engineering helps to diversify the production of enzymes or increase the amount produced by organisms. The complete enzymatic hydrolysis uses cellulolytic enzymes: endoglucanases, exoglucanases and $\beta$-glucosidases, hardly produced in industrially interesting amounts by a single organism, being necessary to combine several of them. In addition, techniques such as recycling or recirculation of enzymes within the bioreactor would help an integral use. Another line of research is the mathematical modeling of the production of enzymatic crudes with simulators such as Superpro Designer and others. The great variety of products obtained from plant biomass, from ethanol to citric acid, lactic acid, uronic acids, acetic acid, etc., support the economic, social, industrial and ecological benefits that this technology would generate. 


\section{Introducción}

En la actualidad es imprescindible orientar sabiamente el rumbo del conocimiento, ya que el vertiginoso avance de la ciencia y la tecnología ha colocado a la humanidad en la cúspide del bien y del mal (Valdés et al, 2004). Es preciso conocer mejor los procesos técnicos, su impacto y sus interrelaciones con la evolución económica para que tanto los individuos como los gobiernos pueden comprender e influir en el progreso técnico y encauzarlo (UNESCO, 1977). La ciencia vinculada a los problemas sociales y convertida en una fuerza productiva basada en la generación de conocimientos para la búsqueda de soluciones a estos problemas, será la base que pueda asegurar un desarrollo sostenible a mediano plazo y pueda además resolver la problemática del desarrollo (Concepción y González, 2013). La ciencia, y las producciones de la ciencia, deben ocupar algún día el primer lugar de la economía nacional (Castro, 1993).

El presente trabajo parte de los desechos lignocelulósicos que se producen durante la actividad agrícola. Es necesario recordar que la biomasa constituye la materia prima más cuantiosa del planeta. Se considera que desempeña el mismo papel que el petróleo lo hizo en el siglo XX (Sims, 2010), pero pese a que se han realizado varios estudios sobre su manejo aún no se encuentra una aplicación práctica significativa (Mesa, Morales, Castro y Cara, 2009).

La biomasa vegetal representa una de las fuentes más importantes sostenibles de combustibles orgánicos, productos químicos y materiales (Lynd, Wiman y Gerngross, 1999). Este es un subproducto de enorme disponibilidad que se genera a partir de diferentes fuentes, entre ellas las agroindustriales (Alvarez et al, 2012). La utilización de la biomasa celulósica sigue siendo un tema de interés mundial en vista del agotamiento rápido del petróleo y de las altas posibilidades químicas energéticas de esta materia prima (Nwodo-Chinedu y Okochi, 2011).

La bioconversión de la lignocelulosa se está estudiando como un medio para aliviar la escasez de los alimentos, la energía y la reducción de la contaminación (Nwodo-Chinedu et al, 2007). Sin embargo la conversión de biomasa basada solo en la utilización de celulosa tiene pocas oportunidades de volverse económicamente atractiva (Ghose y Bisaria, 1987).

La hidrólisis enzimática de la celulosa está basada en la habilidad de hongos y bacterias de producir un set de enzimas extracelulares capaces de degradar las moléculas de D-glucosa (Albernas et al, 2014) que componen la estructura de la celulosa. La hidrólisis enzimática va ganando terreno (Morales, Verelst, Mesa y González, 2014), debido a que la hidrólisis ácida empleada para la obtención de azúcares fermentables ha generado algunos problemas en cuanto a costo y rendimiento (Sarouh, Jover y González, 2005).
Aunque un gran número de microorganismos (hongos, bacterias y actinomicetos) son capaces de degradar la celulosa, como los microhongos celulolíticos pertenecientes a los géneros Trichoderma y Aspergillus que son productores de celulasas notables (De Vries y Viser, 2005), lastimosamente sólo unos pocos de ellos producen cantidades significativas de fracciones de enzimas libres capaces de la hidrólisis completa de la celulosa in vitro. La búsqueda continua de organismos altamente secretadores de enzimas celulolíticas para optimizar la producción (De Vries y Viser, 2005) es una prioridad de la Biotecnología industrial aplicada a la obtención de azúcares reductores. La comprensión de estos microorganismos no sólo juega un papel muy importante por sus aplicaciones industriales sino en el proceso de reciclaje de varios productos como la celulosa en la biosfera (Han, Feng, Zhu y Zhang, 2009).

En las diferentes reacciones químicas que se producen para descomponer los desechos lignocelulósicos, se generan una serie de azúcares y otros componentes cuya fermentación presenta ciertas dificultades, y se podrían necesitar microorganismos modificados genéticamente o mutados (Mesa, Gonzáles, Cara, Ruiz, Castro, y Mussatto, 2010). Al respecto, del análisis de la diversidad genómica de algunos microorganismos empleados para la obtención de celulasas, existe una pobre presencia de genes codificadores de actividad celulolítica; un ejemplo lo constituye el genoma de $T$. reesei, que ha revelado un repertorio inesperadamente bajo de genes de celulasas y hemicelulasas (Foreman et al, 2003). Además, la capacidad celulolítica sobre residuos lignocelulósicos de la mayoría de microorganismos, por ejemplo de hongos en estado natural, no se expresa en condiciones de laboratorio (Herrera García, 2011). Esto, a su vez, repercute en la carencia de biocatalizadores eficientes tolerables a las condiciones de laboratorio e industriales para la producción de enzimas de interés, pues las enzimas muestran una baja actividad y, por lo tanto, son consumidas de forma elevada (Barriga, 2011).

Estas características complican la tarea investigativa para algunos científicos y/o productores de bioetanol y derivados de éste, debido a que frecuentemente emplean ejemplares clásicos de celulasas de origen microbiano, descritas en numerosos trabajos publicados en la segunda mitad del siglo XX, que no son tan eficientes (Gusakov, 2011). Por estas razones, en los últimos años, los investigadores y, sobre todo, las empresas productoras de enzimas han creado organismos recombinantes para la producción heteróloga de celulasas o han mejorado mediante mutaciones los organismos preexistentes.

También se realizan investigaciones para buscar nuevas enzimas, más eficientes y menos costosas en la microbiota simbionte de animales como la lombriz de tierra (Salvador et al, 2012; Salvador, Destain, Rojas, Vásquez y Paz-y-Miño, 2011), debido a que algunos seres vivientes son reconocidos por tener en 
el interior de su cuerpo microorganismos degradadores de un sinnúmero de sustancias con las que éstos entran en contacto de acuerdo al nicho ecológico que realizan; esto hace que ellos sean considerados biorreactores naturales, tal es el caso de las termitas, animales rumiantes, cangrejos de río, y otros. Por ejemplo, las lombrices de tierra son conocidas por ser vectores para la dispersión de microorganismos del suelo que producen enzimas como las amilasas, lipasas, proteasas, etc., y fermentadores para ciertos géneros de microorganismos (Lynd, Weimer, van Zyl y Pretorius, 2002). Algunas especies de lombriz pueden participar en la descomposición de lignina y en los procesos de humificación con las peroxidasas (Brown, Barois y Lavelle, 2000), lo cual resulta interesante por la gran dificultad con la que se descomponen los xilanos. Es importante señalar que el intestino de este animal tiene mecanismos de regulación de temperatura, de humedad, alta concentración de moco que puede facilitar la degradación; además, el estómago es un molino coloidal en el cual el alimento es transformado en pequeñas partículas de $2 \mathrm{~mm}$, lo que da una mejor superficie para el trabajo que hacen los microorganismos. Se ha constatado una presencia elevada de enzimas nitrato reductasas, celobiasas, endoglucanasas fosfatasas ácidas en los intestinos de lombriz. Modelos animales como éste podrían ayudar a vislumbrar nuevas alternativas de degradación de compuestos a partir de procesos naturales eficientes.

También las herramientas modernas de metagenómica, interactoma, metabolómica y pirosecuenciación logran estudiar los diferentes genomas degradadores, existentes en los intestinos del animal, que pueden ser altamente eficientes en la hidrólisis enzimática; el coste económico de éstas tecnologías es bastante alto, sin embargo.

Las enzimas para degradar la biomasa celulósica son compuestos degradadores. Pertenecen a las proteínas de forma globular, funcionan como unidades funcionales del metabolismo celular y actúan acelerando reacciones químicas biológicas como reguladoras de rutas metabólicas. Para actuar necesitan $\mathrm{pH}$ y temperatura óptimos. Existen enzimas sintéticas y también enzimas de origen microbiológico. El presente trabajo estudia las de origen microbiológico que son capaces de degradar celulosa mediante hidrólisis.

Para la hidrólisis enzimática completa es necesaria la actividad de tres enzimas celulolíticas; endoglucanasas y exoglucanasas suplementadas con B-glucosidasas cuyo objetivo es asegurar la ruptura de las moléculas de celobiosa generadas por la acción de las otras enzimas (Breuil, Chan y Saddler, 1990). Al respecto, es difícil que un solo microorganismo de forma natural pueda producir las enzimas celulolíticas necesarias para una degradación exitosa. Es importante la acción sinérgica de varios microorganismos (Ryu y Mandels, 1980). Esto también ha conllevado a la necesidad de crear microorganismos modificados genéticamente (Mesa et al, 2010), aprovechando herramientas tecnológicas que se conocieron en la década de los 70, con el descubrimiento realizado por Stanley Cohen y Herbert Bayer que emplean el aislamiento e inserción de un genoma a otro (Antunes, Pereira y Ebole, 2004). Entonces se reprograman organismos productores de enzimas celulolíticas para obtener cantidades altas de las enzimas en estudio.

Pese a que con el advenimiento de la genética moderna los costos de producción de azúcares a partir de estas fracciones recalcitrantes se han reducido significativamente (Adsul et al, 2004; Kovacs, Szakacs y Zacch, 2009), aún el proceso no es eficiente a escala industrial.

Para reducir el coste de las enzimas se pueden investigar nuevas fuentes de enzimas y/o se pueden reciclar las enzimas utilizando su relativa alta estabilidad y su alta afinidad por la celulosa. Al respecto, a nivel mundial e Iberoamericano, representando el $50 \%$ y 53\% bajo el código de clasificación C12N, relacionado al estudio de microorganismos o enzimas, se encuentran clasificadas más de la mitad de patentes (Red de Indicadores de Ciencia y Tecnología, 2015), como se observa en el Figura 1.

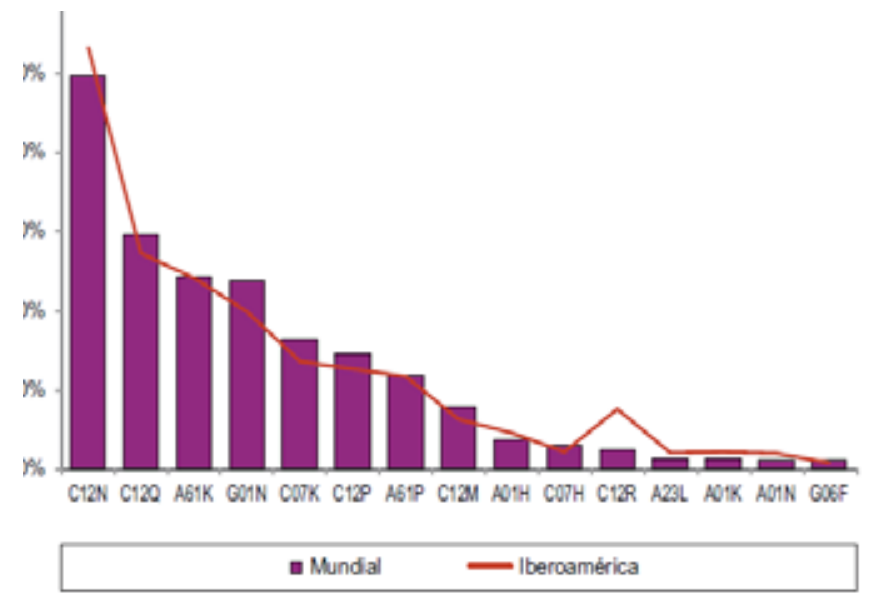

Figura 1. Principales códigos de clasificación (4 dígitos) en el mundo e lberoamérica para Biotecnología (acumulado 2007-2013).

Resulta prioritario estudiar la interacción de las enzimas que degraden lignina (lacasas), celulosa (celulasas y xilanasas) para mejorar el proceso de degradación biomásica.

Para convertir los desechos lignocelulósicos a etanol y otros coproductos, es preciso transformar la estructura polimérica en azúcares sencillos, mediante un pretratamiento de la biomasa lignocelulósica. Además, para asegurar un aprovechamiento integral de los materiales resultantes es necesario utilizar por separado las tres fracciones principales del bagazo (celulosa, hemicelulosa y lignina). En este contexto surge el concepto de fraccionamiento de la biomasa, que como consecuencia deriva en el novedoso campo de la biorrefinería; un ejemplo constituye no solamente la obtención de etanol a partir de glucosa y celobiosa, sino de ácido cítrico, ácido láctico, iso- 
butanol, etc. También mediante conversión catalítica de la fracción hemicelulósica se obtienen pentosas, hexosas y ácidos urónicos, ácido acético, hidroximetilfurfural; de las pentosas se obtienen xilanos, ácido fórmico, furfural, etc (Mesa et al, 2010)

\section{Metodología y resultados}

El presente estudio nace del problema de la carencia de una tecnología asequible, rentable y limpia para el aprovechamiento de residuos lignocelulósicos en el proceso de obtención de etanol y derivados de los procesos fermentativos de los diferentes azúcares y subproductos que se obtienen a partir de la degradación de la celulosa. En este contexto, el empleo de crudos enzimáticos podría definir una disminución del coste de la tecnología y un incremento de los productos que se desean obtener.

El objetivo es la búsqueda de nuevos crudos enzimáticos de origen microbiológico, que permitan aprovechar la capacidad celulolítica de hongos y bacterias existentes en material en degradación de bosques y residuos agrícolas, en una Biorrefinería. En este proceso, la caracterización de las fuentes enzimáticas, su optimización en el proceso de hidrólisis y la recirculación de las mismas es un objetivo prioritario, debido además a que la biomasa lignocelulósica también tiene enzimas (Mussato y Teixeira, 2010). En este ámbito científico y tecnológico se generan nuevos crudos enzimáticos a partir de técnicas biotecnológicas tradicionales y modernas y se genera información sobre los mecanismos de acción de las enzimas estudiadas.

Para complementar el enfoque tecnológico se recomienda estudiar factores como velocidad inicial, características estructurales como el área superficial, el grado de acumulación de agua, el orden molecular, la estructura capilar de las fibras de celulosa, el contenido de materiales asociados y la cristalinidad (Herrera García, 2011; Ramos, Breuil, Kushner y Saddler, 1992) También han sido estudiados mecanismos de recirculación de las enzimas en los procesos de hidrólisis enzimática y fermentación.

Es frecuente que la hidrólisis enzimática ya no vaya separada de la fermentación (HFS), sino que se realice en un solo esquema, que consiste en la sacarificación y fermentación simultáneas (SFS) del residuo (Ballesteros, Oliva, Negro, Manzanares, y Ballesteros, 2004). El proceso a emplear está en dependencia de los biocatalizadores y/o enzimas que se emplearán. En estos procesos se forma una mezcla sólido-líquido y las enzimas que se encuentran en la fase líquida pueden recircularse añadiendo sustrato fresco; a las enzimas que se encuentran en la fase sólida se les añade soluciones tamponadas para su recuperación (Barriga, 2011). La recirculación puede optimizar la fermentación y reducir los costes de la producción de la enzima, que es uno de los objetivos de la investigación básica para aplicaciones industriales (HoaPham, Quyen, y Nghiem, 2010).
La recirculación de las enzimas es otra alternativa para contribuir a solucionar el problema del alto coste de las enzimas. Para recircular se necesita conocer parámetros específicos como: factores de inhibición del producto, desactivación térmica, mecánica y depleción del componente celulósico específico por adsorción con pérdida de energía cinética, etc (Barriga, 2011). Estos parámetros podrían ayudar a mejorar la eficiencia de la hidrólisis, que decrece gradualmente en cada paso de reciclado (Mussato y Teixeira, 2010).

La tecnología a emplear en el presente estudio incluye un estudio de vigilancia tecnológica. Además cuenta con la modelación matemática de los balances de masa y energía para predecir la producción de los crudos enzimáticos con simuladores como Superpro Designer, para determinar las mejores condiciones de obtención del producto con capacidad degradadora para posterior verificación experimental. El establecimiento de una propuesta tecnológica y experimental se basa en los diseños de Plackett-Burman y en el programa Design Expert para comprender los parámetros que afectan la producción enzimática, los tipos de enzimas producidas y la recirculación de las mismas.

Los impactos que generará la propuesta tecnológica no son solamente del orden científico-técnico como se ha explicado, sino económico, social, productivo y ambiental. Ambientalmente, la materia prima empleada no compite con biomasa alimenticia. Además de reducir el volumen de desecho en un 40\%, los problemas de tratamiento, gestión y riesgo de desechos urbanos, agrícolas y forestales mejoran. La propuesta contribuye a prevenir el cambio climático mundial, mitigar los gases de efecto invernadero, disminuir los lixiviados que contaminan las aguas subterráneas, entre otros efectos. Previene los graves problemas del deterioro del suelo consecuencia de la deforestación, limitando los avances de la frontera ganadera y agrícola que tanto ha simplificado los ecosistemas (Funes, 2005), lo que a su vez influye en la reducción de precipitaciones y en la desaparición de numerosas especies vegetales y animales. Esto ayuda a que se cumpla el Objetivo 12 de Desarrollo Sostenible de las Naciones Unidas, que es el de Garantizar Modalidades de Consumo y Producción Sostenibles (Organización de las Naciones Unidas [ONU]: Objetivos de Desarrollo Sostenible de las Naciones Unidas. Juntos por el Desarrollo, 2016).

A nivel social, el empleo de materiales reciclados, que nos permitan tener una vida dentro de lo que se considera sostenible, sustentable para crear ciudades resilientes que puedan retroalimentarse y autoabastecerse, es un tema que ha sido debatido y propuesto en la reunión de Habitat III (ONU, 2017). Además, permite la generación de nuevos productos y materias primas.

De ser implementados en Ecuador los resultados de la presente propuesta, se promovería el crecimiento 
económico sostenido, inclusivo y sostenible en el que se incrementa el trabajo mediante el empleo pleno y productivo y el trabajo decente para todos, que también es objetivo de las Naciones Unidas para el Desarrollo Sostenible.

A nivel productivo, el manejo óptimo de desechos, que puede converger con otras industrias, impulsará indirectamente el desarrollo de la matriz productiva y energética de nuestros países, lo que a nivel económico disminuye el coste de producción y por lo tanto incrementa la rentabilidad de la inversión.

El presente trabajo también contribuye a favorecer las políticas públicas a favor del reciclado y el uso de materiales reciclados por parte de las diferentes empresas productivas.

\section{Conclusiones}

El presente estudio responde a la necesidad de tecnología accesible, rentable y limpia para el aprovechamiento de residuos lignocelulósicos en el proceso de obtención de etanol y derivados de los procesos fermentativos de los diferentes azúcares y subproductos que se obtienen a partir de la degradación de la celulosa. El empleo de crudos enzimáticos podría efectivamente disminuir del coste de la tecnología y aumentar el volumen de los productos que se deseen obtener.

El establecimiento de una tecnología enzimática mediante la incorporación al diseño industrial de una tecnología de producción in situ de enzimas para descomponer los desechos biomásicos en un contexto de diversificación del producto es una propuesta interesante por la generación de nuevos catalizadores e instalaciones tecnológicas que respondan a las necesidades técnicas y sociales.

La ejecución de un proyecto de la magnitud y trascendencia planteada requiere del trabajo colaborativo de la comunidad científica, aunando experiencias, conocimiento y capacidades para alcanzar los objetivos planteados.

\section{Referencias bibliográficas}

Adsul, M.G., Ghule, J.E., Singh, R., Shaikh, H., Bastawde, K. B., Gokhale, D.V., y Varma, A.J. (2004). Polysaccharides from bagasse: applications in cellulase and xylanase production. Carbohydrate Polymers, 57, 1, 67-72. doi:10.1016/j.carbpol.2004.04.001.

Albernas-Carvajal, Y., Corsano, G., Morales-Zamora, M., González-Cortés, M., Santos-Herrero, R., y González-Suárez, E. (2014). Optimal design for an ethanol plant combining first and second-generation technologies. CT\&F - Ciencia, Tecnología y Futuro, 5 (5), 97-120.

Álvarez Castillo, A., García Hernández, E., Domínguez Domínguez, M.M., Granandos Baeza, J.M., Aguirre Cruz, A., Carmona García, R., Mendoza Martínez,
A.M. (2012). Aprovechamiento integral de los materiales lignocelulósicos. Revista Iberoamericana de Polímeros, 13 (4), 141-150.

Antunes, A., Pereira, N., y Ebole, M. F. (2006). Gestão em Biotecnologia. (1ra. Ed.) Rio de Janeiro: E-papers.

Ballesteros, M., Dominguez, J., Negro, M., Manzanares, P., y Ballesteros, I. (2004). Ethanol from lignoceIlulosic materials by a simultaneous saccharification and fermentation process (SFS) with Kluyveromyces marxianus CECT 10875. Process Biochemistry, 39. 1843-1848. doi: 10.1016/j.procbio.2003.09.011.

Barriga, D. (2011). Posibilidades de recirculación de enzimas celulolíticas en la hidrólisis del bagazo de caña de azúcar. (Trabajo de Diploma). Facultad de Ciencias Agropecuarias. Universidad Central Marta Abreu de Las Villas, Cuba.

Breuil, C., Chan M., y Saddler, J. N. (1990). Comparison of the hydrolytic activity of commercial cellulase preparations. Applied Microbiology Biotechnology. 34, 31-35. doi: 10.1007/BF00170919

Brown, G., Barois, I., y Lavelle, P. (2000). Regulation of soil organic matter dynamics and microbial activity in the drilosphere and the role of interactions with other edaphic functional domains. European Journal of Soil Biology, 36, 177-198. doi: 10.1016/S11645563(00)01062-1.

Castro, F. (1993) Discurso pronunciado durante la Inauguración del Centro de Biofísica Médica.

Concepción, D. y González, E. (2013). La gestión del conocimiento en el vínculo universidad- empresa para el desarrollo local y territorial. V Conferencia de la Ciencias Sociales y Humanísticas.

De Vries R. P. y Viser, J. (2001). Aspergillus enzymes involved in degradation of plant cell wall polysaccharides. Microbiol. Mol. Biol. Rev. 65, 497-552.

Foreman, P. K. (2003). Transcriptional regulation of biomass degrading enzymes in the filamentous fungus Trichoderma reesei. J. Biol. Chem. 278, 3198831997.

Funes R. (2005). História Ambiental na América Latina. Belo Horizonte. Varia Historia. Vol 21.33

Ghose, T., y Bisaria, V. (1987). Measurement of Hemicellulase Activities. Pure \& Appl. Chem. Vol. 59, No.12, pp. 1739-1752. Great Britain. Part 1: Xylanases.

Gusakov A. V. (2011). Alternatives to Trichoderma reesei in biofuel production. Cell Press. Department of Chemistry, M. V. Lomonosov Moscow State University, Vorobyovy Gory 1/11, Moscow 119991, R.

Han, L., Feng, J., Zhu, C. y X. Zhang. (2009). Optimizing cellulase production of Penicillium waksmanii 
F10-2 with response surface methodology. African Journal of Biotechnology. 8 (16), pp. 3879-3886.

Herrera-García, M. (2011). Capacidad celulolítica de hongos existentes en la naturaleza para degradar residuos lignocelulósicos. (Tesis de diploma). Facultad de Ciencias Agropecuarias. Universidad Central Marta Abreu de Las Villas, Cuba.

Hoa P., Thi, Q., Nghiem, N. (2010). Optimization of endoglucanase production by Aspergillus niger VTCC-F021. Australian Journal of Basic and Applied Sciences. 6. 4151-5157.

Kovacs, K., Szakacs, G., Zacch, G.(2009). Enzymatic hydrolysis and simultaneous saccharification and fermentation of steam-pretreated spruce using crude Trichoderma reesei and Trichoderma atroviride enzymes. Process Biochemistry. 44, 1323-1329

Lynd, L. R., Wyman, C. E., y Gerngross, T. U. (1999). Biocommodity engineering. Biotechnol. Prog. 15, 777-793.

Lynd, L.R., Weimer, P. J., van Zyl, W. H., y Pretorius, I. (2002). Microbial Cellulose Utilization: Fundamentals and Biotechnology. Microbiol. Mol. Biol. Rev., 66 (3), 506.

Mesa, L, González, E., Morales, M., Castro, E., Cara, C., y Kafarov, V. T., (2009). Technico-Economic Evaluation of Alternatives for Assimilation of ethanol production technology from sugar cane bagasse. Congreso de Medio Ambiente y Biocombustibles. Dubrovnik, Croatia.

Mesa, L., González, E., Cara, C., Ruiz, E., Castro, E., y Mussatto, S. (2010). An approach to optimization of enzymatic hydrolysis from sugarcanne bagasse based on organosolv pretreatment. Journal Technical Bioetchnology. 85, 1092-1098.

Morales, M., Verelst, H., Mesa, L., y González, E. (2010). Simulation of furfural production process for revamping with ethanol technology from lignocellulosic residuals. Chemical Engineering Transactions. 21, 967-972. doi: 103303/CET 1021162.

Mussato, S.I., y Teixeira, J. A. (2010). Lignocelulose as raw material in fermentation processes, $\mathrm{Cu}$ rrent Research, Technology and Education Topics. Applied Microbiology and Microbial Biotechnology, 2, 897-907.

Nwodo-Chinedu, S. y Okochi, V. I. (2011). Cellulase Production by wild-type Aspergillus niger, Penicillium chrysogenum and Trichoderma harzianum using waste cellulosic materials. Journal of Science. 13, no. 1

Nwodo-Chinedu, S., Okochi, V. I., Smith, H.,Okafor, U., Onyegeme-Okerenta, B. M. y Omidiji, O.(2007). Effect of carbon sources on cellulase (EC 3. 2. 1. 4) production by Penicillium chrysogenum PCL50. Afri- can Journal of Biochemistry Research. 1 (1), 006-010.

Organización de las Naciones Unidas, ONU. (2016) Objetivos de Desarrollo Sostenible de las Naciones Unidas. Juntos por el Desarrollo.

Organización de las Naciones Unidas, ONU. (2017) Nueva Agenda Urbana. Conferencia de las Naciones Unidas sobre la Vivienda y el Desarrollo Urbano Sostenible (Hábitat III). Recuperado de http://habitat3. org/the-new-urban-agenda

Ramos, L. P., Breuil, C., Kushner, D. J. y Saddler, J. N. (1992). Steam pretreatment conditions for effective enzymatic hydrolysis and recovery yields of Eucalyptus viminalis wood chips. Holzforschung - International Journal of the Biology, Chemistry, Physics and Technology of Wood, 46(2), 149-154. doi:10.1515/ hfsg.1992.46.2.149

RICYT. (2015). El Estado de la Ciencia. Principales Indicadores de Ciencia y Tecnología Iberoamericanos/Interamericanos. Red de indicadores de Ciencia y Tecnología.

Ryu, D. y Mandels, M. (1980). Cellulases: Biosynthesis and applications. Enzyme and Microbial Technology. 2. 91-102. doi: 10.1016/0141-0229(80)90063-0

Salvador, C. A., Destain, J., Rojas, M., Vásquez, E., y Paz-y-Miño, C. (2011). Producción de actividades enzimáticas por el intestino de Eisenia foetida (Annelida: Clitellata: Haplotaxida) Revista Ciencia. 14, 2 , 191-198

Salvador, C. A., Rojas, M., Jaramillo-Kouperman, G., Yépez, L., Suárez, J. P., Mesa, L., y Paz-y-Miño, C. (2012). Búsqueda de bacterias con actividad EC 3.2.1.4 (endo-1,4- beta-glucanasa) en Eisenia foetida (Oligochaeta, Lumbricidae). Revista Ecuatoriana de Medicina y Ciencias Biológicas.

Sarrouh, B. F., Jover, J., y González, E. (2005). Estudio de la hidrólisis del bagazo con ácido sulfúrico concentrado utilizando dos variantes de una sola etapa y una sola etapa modificada para la obtención de etanol y análisis técnico-económico de dicho proceso. Ingeniería e Investigación, 25 (3), 34-38. Recuperado de http://www.scielo.org.co/scielo.php?script=sci_ arttext\&pid=S0120-56092005000300005\&lng=en\&tl$\mathrm{ng}=\mathrm{es}$.

Sims, R.E.H. (2010). An overview of second generation biofuel technologies. Bioresour. Technol.. 101, 1570-1580.

UNESCO.(1997). Simposio sobre las repercusiones sociales de la revolución científica y tecnológica.

Valdés, C., Hernández, L., Pimentel, L., López, N., Flores, M.. (2004). Problemas Sociales de la Ciencia y la Tecnología. Selección de lecturas. La Habana, Cuba: Editorial Félix Varela. 